\title{
Approximate solutions and error estimates for a Stokes boundary value problem
}

\author{
Frank Müller ${ }^{1}$ \\ ${ }^{1}$ Berufsakademie Nordhessen \\ University of Cooperative Education \\ Eichlerstraße 25 \\ D-34537 Bad Wildungen \\ Tel: +49-5621-9658817 \\ f.mueller@ba-nordhessen.de
}

\author{
Werner Varnhorn ${ }^{2}$
}

\author{
${ }^{2}$ Universität Kassel \\ Heinrich-Plett-Str. 40 (AVZ) \\ D-34132 Kassel \\ Tel: +49-561-8044614 \\ Fax: +49-561-8044443 \\ varnhorn@mathematik.uni-kassel.de
}

The aim of this paper is the numerical treatment of a boundary value problem for the system of Stokes' equations. For this we extend the method of approximate approximations to boundary value problems. This method was introduced by V. Maz'ya in 1991 and has been used until now for the approximation of smooth functions defined on the whole space and for the approximation of volume potentials.

In the present paper we develop an approximation procedure for the solution of the interior Dirichlet problem for the system of Stokes' equations in two dimensions. The procedure is based on potential theoretical considerations in connection with a boundary integral equations method and consists of three approximation steps as follows.

In a first step the unknown source density in the potential representation of the solution is replaced by approximate approximations. In a second step the decay behavior of the generating functions is used to gain a suitable approximation for the potential kernel, and in a third step Nyström's method leads to a linear algebraic system for the approximate source density. For every step a convergence analysis is established and corresponding error estimates are given. 


\section{Introduction}

In $1991 \mathrm{~V}$. Maz'ya proposed a new approximation method called the method of approximate approximations [9], which is based on generating functions representing an approximate partition of unity, only. As a consequence, this approximation method does not converge if the mesh size tends to zero.

For practical computations this lack of convergence does not play an important role since the resulting error can be chosen less than machine precision. On the other hand, this method has great advantages due to nice properties of the generating functions, i.e. simplicity, smoothness and exponential decay behavior [11].

The method of approximate approximations can be used efficiently for the evaluation of various problems in mathematical physics, e.g. Cauchy problems of the kind $L u=f$, where $L$ denotes a suitable linear differential operator in $\mathbb{R}^{n}$. Approximating the right hand side $f$ by approximate approximations, in many cases explicit formulas for the approximating volume potentials are obtained containing a one-dimensional integration, only.

For boundary value problems, the method of approximate approximations has been applied, too: For example, there are some heuristic considerations concerning the so-called boundary point method [10] and, moreover, some detailed results about the Dirichlet problem for the Laplace equation $[12,13]$.

In the present paper we develop an approximation method for the solution of the interior Dirichlet problem for the system of Stokes' equations in two dimensions using approximate approximations. In hydrodynamics the system of Stokes' equations describe the stationary flow of a viscous incompressible fluid with low velocity. Solutions to the system of Stokes' equations consist of vector field describing the velocity and a scalar function describing the pressure. The velocity field is called Stokes function.

The procedure is based on potential theoretical considerations in connection with a boundary integral equations method and consists of three approximation steps as follows:

In a first step the unknown source density in the potential representation of the solution is replaced by approximate approximations. In a second step the decay behavior of the generating functions is used to gain a suitable approximation for the potential kernel, and in a third step Nyström's method leads to a linear algebraic system for the approximate source density. For every step a convergence analysis is established and corresponding error estimates are given.

The method, which is explicitly carried out here for the interior Dirichlet problem of the Stokes operator in two dimensions, can also be used for many other boundary value problems in mathematical physics, whenever a suitable potential theory is available, as it is e.g. in the case of harmonic, elastic, or hydrodynamic boundary value problems in general space dimensions. In all these cases there exists also a representation of the 
solution in form of a boundary layer potential, where the unknown source density has to be determined as a solution of Fredholm boundary integral equations and can be approximated by the method developed here, too.

\section{The Dirichlet problem}

Let $G \subset \mathbb{R}^{2}$ be a bounded simply connected domain with boundary $\Gamma$ of class $C^{2}$. We consider the following Dirichlet problem for the system of Stokes equations: Find a velocity field $v \in C^{2}\left(G, \mathbb{R}^{2}\right) \cap C\left(\bar{G}, \mathbb{R}^{2}\right)$ and a pressure function $p \in C^{1}(G)$ solving

$$
-\Delta v+\nabla p=0 \quad \text { in } G, \quad \nabla \cdot v=0 \quad \text { in } G, \quad v=b \quad \text { auf } \Gamma .
$$

Here the Laplacian $\Delta$ is applied to the components $v_{1}$ and $v_{2}$ of the velocity field $v=$ $\left(v_{1}, v_{2}\right)^{T}, \nabla p=\left(\partial_{1} p, \partial_{2} p\right)^{T}$ denotes the gradient of $p$ and $\nabla \cdot v=\partial_{1} v_{1}+\partial_{2} v_{2}$ is the divergence of $v$. Furthermore $b \in C\left(\Gamma, \mathbb{R}^{2}\right)$ is a given boundary value. Using the Gaussian integral theorem and the fact that $v$ has no divergence, we get the following condition which is necessary for the existence of a solution

$$
\int_{\Gamma} b(y) \cdot n(y) d s(y)=0 .
$$

Here $n(y)$ denotes the outward unit normal in $y \in \Gamma$.

From hydrodynamical potential theory it is known [14], [15], [17]: If the condition (1) is fulfilled, the the Dirichlet problem has a solution $(v, p)$. The velocity field $v$ is uniquely determined and the pressure function $p$ is uniquely determined up to an additive constant.The velocity field $v$ can be represented in $G$ by the hydrodynamical double layer potential

$$
(D \varphi)(x):=\int_{\Gamma} d(x, y) \varphi(y) d s(y), \quad x \in G .
$$

Here the vector field $\varphi=\left(\varphi_{1}, \varphi_{2}\right)^{T}: \Gamma \rightarrow \mathbb{R}^{2}$ is an unknown continuous density and the kernel $d(x, y)$ of the hydrodynamical double layer potential for $x \in \mathbb{R}^{2}$ and $y \in \Gamma$ with $x \neq y$ is defined by the $2 \times 2$ matrix

$$
d(x, y):=-\frac{(x-y) \cdot n(y)}{\pi|x-y|^{4}}\left(\begin{array}{cc}
\left(x_{1}-y_{1}\right)^{2} & \left(x_{1}-y_{1}\right)\left(x_{2}-y_{2}\right) \\
\left(x_{1}-y_{1}\right)\left(x_{2}-y_{2}\right) & \left(x_{2}-y_{2}\right)^{2}
\end{array}\right) .
$$

Under the required regularity assumptions on the boundary $\Gamma$ it follows [8] that the kernel of the hydrodynamical double layer potential can be continuously extended to $\Gamma \times \Gamma$. So there exists for every $x \in \Gamma$ the so-called direct value

$$
(D \varphi)(x):=\int_{\Gamma} d(x, y) \varphi(y) d s(y), \quad x \in \Gamma .
$$


Using the jump relations for the hydrodynamical double layer potential [8] we get that the unknown density $\varphi$ has to be determined as a solution the Fredholm boundary integral equation system of second kind

$$
\frac{1}{2} \varphi(x)+(D \varphi)(x)=b(x), \quad x \in \Gamma,
$$

where $(D \varphi)(x)$ here denotes the direct value.

For every $b \in C\left(\Gamma, \mathbb{R}^{2}\right)$ which fulfills the condition (1) this Fredholm boundary integral equation system of second kind has a solution $\varphi \in C\left(\Gamma, \mathbb{R}^{2}\right)$, which is not uniquely determined [1], [17]. For numerical purposes we are interested in uniquely solvable boundary integral equation systems, since after a suitable discretisation these systems lead to a uniquely solvable linear algebraic system. To establish uniqueness of the solution we define the operator

$$
N: C\left(\Gamma, \mathbb{R}^{2}\right) \rightarrow C\left(\Gamma, \mathbb{R}^{2}\right), \quad \varphi \mapsto N \varphi
$$

with

$$
(N \varphi)(x):=n(x) \int_{\Gamma} \varphi(y) \cdot n(y) d s(y)
$$

and instead of the above boundary integral equation system consider the following system

$$
\frac{1}{2} \varphi(x)+(D \varphi)(x)-(N \varphi)(x)=b(x), \quad x \in \Gamma .
$$

It can be shown [1], [17] that for every $b \in C\left(\Gamma, \mathbb{R}^{2}\right)$ this system has a unique solution and that this solution is also a solution of the initial system if the condition (1) is fulfilled. In the following we will always consider the uniquely solvable system (2).

Let $\gamma:[-1,1] \rightarrow \Gamma$ be a parameterization of the boundary $\Gamma$. Then we obtain both in $x \in G$ for the hydrodynamical double layer potential and in $x \in \Gamma$ for its direct value the representation

$$
(D \varphi)(x)=\int_{-1}^{1} d(x, \gamma(t)) \varphi(\gamma(t))\left|\gamma^{\prime}(t)\right| d t .
$$

Setting

$$
u:=\varphi \circ \gamma
$$

and

$$
K(x, t):=D(x, \gamma(t))\left|\gamma^{\prime}(t)\right|,
$$

for the hydrodynamical double layer potential in $G$ and for the direct value on the boundary $\Gamma$ we get the representation

$$
\begin{aligned}
(D \varphi)(x) & =\int_{-1}^{1} K(x, t) u(t) d t \\
& =\int_{-1}^{1}\left(\begin{array}{l}
k_{11}(x, t) u_{1}(t)+k_{12}(x, t) u_{2}(t) \\
k_{21}(x, t) u_{1}(t)+k_{22}(x, t) u_{2}(t)
\end{array}\right) d t .
\end{aligned}
$$




\section{The approximation procedure}

As mentioned above, in $G$ the uniquely determined velocity field can be represented by the hydrodynamical double layer potential, i.e. we have

$$
v(x)=\int_{-1}^{1} K(x, t) u(t) d t, \quad x \in G .
$$

In the following we will approximate the velocity field $v$ for $x \in G$ by an explicit analytic expression containing no integrals. This will be done in three steps. To do so, let $N \in \mathbb{N}$, $h:=1 / N, d>0$.

\subsection{The first approximation step}

In the first step we replace the components $u_{i}$ of the unknown function $u$ in the integral representation (3) by the approximate approximations

$$
\left(u_{i}\right)_{d, h}:[-1,1] \rightarrow \mathbb{R}, \quad\left(u_{i}\right)_{d, h}(t):=\frac{1}{\sqrt{\pi d}} \sum_{m=-N}^{N} u_{i}(m h) e^{-\frac{(t-m h)^{2}}{d h^{2}}}
$$

and define for $x \in G$

$$
\Psi_{d, h}(x):=\int_{-1}^{1} K(x, t)\left(\begin{array}{c}
\left(u_{1}\right)_{d, h}(t) \\
\left(u_{2}\right)_{d, h}(t)
\end{array}\right) d t
$$

as an approximation of $v(x)$.

Setting

$$
u_{d, h}(t):=\left(\begin{array}{c}
\left(u_{1}\right)_{d, h}(t) \\
\left(u_{2}\right)_{d, h}(t)
\end{array}\right)=\frac{1}{\sqrt{\pi d}} \sum_{m=-N}^{N} u(m h) e^{-\frac{(t-m h)^{2}}{d h^{2}}}
$$

we get

$$
\begin{aligned}
\Psi_{d, h}(x) & =\int_{-1}^{1} K(x, t) u_{d, h}(t) d t \\
& =\frac{1}{\sqrt{\pi d}} \sum_{m=-N}^{N} \int_{-1}^{1} K(x, t) u(m h) e^{-\frac{(t-m h)^{2}}{d h^{2}}} d t .
\end{aligned}
$$




\subsection{The second approximation step}

Since the function

$$
t \mapsto e^{-\frac{(t-m h)^{2}}{d h^{2}}}
$$

decreases rapidly with increasing distance to $m h$, if the term $d h^{2}$ is sufficiently small, in the second step we replace the kernel $K(x, t)$ by $K(x, m h)$ and define for $x \in G$

$$
\begin{aligned}
\Phi_{d, h}(x) & :=\frac{1}{\sqrt{\pi d}} \sum_{m=-N}^{N} \int_{-1}^{1} K(x, m h) u(m h) e^{-\frac{(t-m h)^{2}}{d h^{2}}} d t \\
& =\frac{1}{\sqrt{\pi d}} \sum_{m=-N}^{N} K(x, m h) u(m h) \int_{-1}^{1} e^{-\frac{(t-m h)^{2}}{d h^{2}}} d t
\end{aligned}
$$

as an approimation for $\Psi_{d, h}(x)$.

Using

$$
\int_{-1}^{1} e^{-\frac{(t-m h)^{2}}{d h^{2}}} d t=\frac{\sqrt{d}}{N} \int_{\frac{m-N}{\sqrt{d}}}^{\frac{m+N}{\sqrt{d}}} e^{-t^{2}} d t=\frac{\sqrt{\pi d}}{2 N} \operatorname{erf}\left(\frac{m-N}{\sqrt{d}}, \frac{m+N}{\sqrt{d}}\right)
$$

we get

$$
\Phi_{d, h}(x)=\frac{1}{2 N} \sum_{m=-N}^{N} K(x, m h) u(m h) \operatorname{erf}\left(\frac{m-N}{\sqrt{d}}, \frac{m+N}{\sqrt{d}}\right),
$$

where for $a, b \in \mathbb{R}$ with $a \leq b$ the error function is defined by

$$
\operatorname{erf}(a, b):=\frac{2}{\sqrt{\pi}} \int_{a}^{b} e^{-t^{2}} d t
$$

\subsection{The third approximation step}

Since the density $\varphi$ is still unknown we do not know the values $u(m h)=\varphi(\gamma(m h))$. Therefore in a third step we determine approximate values $u_{m}$ of $u(m h)$ by using Nyström's method [7]. Since $u=\varphi \circ \gamma$ is the unique solution of the boundary integral equation system (2) we have for all $x \in \Gamma$

$$
\frac{1}{2} \varphi(x)+(D \varphi)(x)-n(x) \int_{\Gamma} \varphi(y) \cdot n(y) d s(y)=b(x),
$$

i.e.

$$
\frac{1}{2} \varphi(x)+\int_{-1}^{1} K(x, t) u(t) d t-n(x) \int_{-1}^{1} u(t) \cdot n(\gamma(t))\left|\gamma^{\prime}(t)\right| d t=b(x) .
$$

So for all $s \in[-1,1]$ we have

$$
\frac{1}{2} u(s)+\int_{-1}^{1} K(\gamma(s), t) u(t) d t-n(\gamma(s)) \int_{-1}^{1} u(t) \cdot n(\gamma(t))\left|\gamma^{\prime}(t)\right| d t=b(\gamma(s)) .
$$


Especially, this system of equations is fulfilled in the points $s=j h$. For $j=-N, \ldots, N$ we then obtain

$$
\frac{1}{2} u(j h)+\int_{-1}^{1} K(\gamma(j h), t) u(t) d t-n(\gamma(j h)) \int_{-1}^{1} u(t) \cdot n(\gamma(t))\left|\gamma^{\prime}(t)\right| d t=b(\gamma(j h)) .
$$

We now require equality if we approximate the integrals by the trapezoidal rule (Nyströms method) and get

$$
\begin{aligned}
b(\gamma(j h))= & \sum_{m=-N}^{N}\left(\frac{2-\delta_{|m| N}}{2 N} K(\gamma(j h), m h)+\frac{1}{2} \delta_{j m}\right) u_{m} \\
& -n(\gamma(j h)) \sum_{m=-N}^{N} \frac{2-\delta_{|m| N}}{2 N} u_{m} \cdot n(\gamma(m h))\left|\gamma^{\prime}(m h)\right| .
\end{aligned}
$$

Here the value $u_{m}=\left(u_{1, m}, u_{2, m}\right)^{T}$ are approximations to the values $u(m h)$ and $\delta_{k l}$ denotes the Kronecker symbol. For the components we find

$$
b_{i}(\gamma(j h))=\sum_{m=-N}^{N}\left(a_{j, m}^{(i 1)} u_{1, m}+a_{j, m}^{(i 2)} u_{2, m}\right), \quad i=1,2
$$

with

$$
\begin{aligned}
a_{j, m}^{(i k):=} & \frac{2-\delta_{|m| N}}{2 N} k_{i k}(\gamma(j h), m h)+\frac{1}{2} \delta_{j m} \delta_{i k} \\
& -\frac{2-\delta_{|m| N}}{2 N} n_{i}(\gamma(j h))\left|\gamma^{\prime}(m h)\right| n_{k}(\gamma(m h)) .
\end{aligned}
$$

These $2(2 N+1)$ equations can be written as the following linear system

$$
\left(\begin{array}{cccccc}
a_{-N,-N}^{(11)} & \ldots & a_{-N, N}^{(11)} & a_{-N,-N}^{(12)} & \ldots & a_{-N, N}^{(12)} \\
\vdots & & \vdots & \vdots & & \vdots \\
a_{N,-N}^{(11)} & \ldots & a_{N, N}^{(11)} & a_{N,-N}^{(12)} & \ldots & a_{N, N}^{(12)} \\
a_{-N,-N}^{(21)} & \ldots & a_{-N, N}^{(21)} & a_{-N,-N}^{(22)} & \ldots & a_{-N, N}^{(22)} \\
\vdots & & \vdots & \vdots & & \vdots \\
a_{N,-N}^{(21)} & \ldots & a_{N, N}^{(21)} & a_{N,-N}^{(22)} & \ldots & a_{N, N}^{(22)}
\end{array}\right)\left(\begin{array}{c}
u_{1,-N} \\
\vdots \\
u_{1, N} \\
u_{2,-N} \\
\vdots \\
u_{2, N}
\end{array}\right)=\left(\begin{array}{c}
b_{1}(\gamma(-1)) \\
\vdots \\
b_{1}(\gamma(1)) \\
b_{2}(\gamma(-1)) \\
\vdots \\
b_{2}(\gamma(1))
\end{array}\right)
$$

for the determination of the $2(2 N+1)$ values $u_{1,-N}, \ldots, u_{1, N}, u_{2,-N}, \ldots, u_{2, N}$.

Finally, for $x \in G$ we set

$$
v_{d, h}(x):=\frac{1}{2 N} \sum_{m=-N}^{N} K(x, m h) u_{m} \operatorname{erf}\left(\frac{m-N}{\sqrt{d}}, \frac{m+N}{\sqrt{d}}\right)
$$

as an approximation for $\Phi_{d, h}(x)$ and hence as an approximation for $v(x)$. 


\section{Convergence analysis}

To investigate the accuracy of the approximation, we define for $x \in G$ the error

$$
F(d, h, x):=\left|v(x)-v_{d, h}(x)\right|
$$

and consider the decomposition

$$
\begin{aligned}
F(d, h, x) & =\left|v(x)-\Psi_{d, h}(x)+\Psi_{d, h}(x)-\Phi_{d, h}(x)+\Phi_{d, h}(x)-v_{d, h}(x)\right| \\
& \leq \underbrace{\left|v(x)-\Psi_{d, h}(x)\right|}_{=: F_{1}(d, h, x)}+\underbrace{\left|\Psi_{d, h}(x)-\Phi_{d, h}(x)\right|}_{=: F_{2}(d, h, x)}+\underbrace{\left|\Phi_{d, h}(x)-v_{d, h}(x)\right|}_{=: F_{3}(d, h, x)},
\end{aligned}
$$

where $F_{i}(d, h, x)$ denotes the error in the $i$-th approximation step.

In the following we assume $b \in C^{2}\left(\Gamma, \mathbb{R}^{2}\right)$. Then we find $\varphi \in C^{2}\left(\Gamma, \mathbb{R}^{2}\right)$ due to the regularizing properties of the double layer potential. Since also $\Gamma$ is of class $C^{2}$ we obtain $\gamma \in C^{2}\left([-1,1], \mathbb{R}^{2}\right)$ and hence $u \in C^{2}\left([-1,1], \mathbb{R}^{2}\right)$.

For the convergence analysis below we need an estimate for the kernel.

For $x \in G$ and $y \in \Gamma$ we have

$$
\left|x_{i}-y_{i}\right| \leq|x-y|, \quad i=1,2
$$

and

$$
|x-y| \geq \operatorname{dist}(x, \Gamma)
$$

where

$$
\operatorname{dist}(x, \Gamma):=\inf _{y \in \Gamma}|x-y|
$$

denotes the distance from $x \in G$ to the boundary $\Gamma$. Using the Cauchy-Schwarz inequality we get

$$
\begin{aligned}
\left|k_{i k}(x, t)\right| & =\frac{\left|(x-\gamma(t)) \cdot n(\gamma(t))\left(x_{i}-\gamma_{i}(t)\right)\left(x_{k}-\gamma_{k}(t)\right)\right|}{\pi|x-\gamma(t)|^{4}}\left|\gamma^{\prime}(t)\right| \\
& \leq \frac{\left\|\gamma^{\prime}\right\|_{\infty}}{\pi|x-\gamma(t)|} \\
& \leq \frac{\left\|\gamma^{\prime}\right\|_{\infty}}{\pi \operatorname{dist}(x, \Gamma)},
\end{aligned}
$$

where $\|\cdot\|_{\infty}$ denotes the supremum norm.

To prove convergence we start with the last step. 


\subsection{Convergence in the third step}

It is known that Nyström's method converges in this setting of at least second order $[3,7]$. Hence for sufficiently large $N$ the linear system given above has a unique solution and there is a constant $C>0$ with

$$
\max _{m \in\{-N, \ldots, N\}}\left|u_{i}(m h)-u_{i, m}\right| \leq C h^{2}, \quad i=1,2 .
$$

This lead to an estimate for the corresponding error in the third step.

Lemma 1. For $F_{3}(d, h, x)$ we have

$$
F_{3}(d, h, x) \leq \frac{3 \sqrt{8} C\left\|\gamma^{\prime}\right\|_{\infty}}{\pi \operatorname{dist}(x, \Gamma)} h^{2}
$$

with the constant $C$ from (5).

Proof. Using the estimates (4), (5) and

$$
\left|\operatorname{erf}\left(\frac{m-N}{\sqrt{d}}, \frac{m+N}{\sqrt{d}}\right)\right| \leq 2
$$

we obtain

$$
\begin{aligned}
F_{3}(d, h, x) & \leq \frac{1}{2 N} \sum_{m=-N}^{N}\left|K(x, m h)\left(u(m h)-u_{m}\right)\right| \operatorname{erf}\left(\frac{m-N}{\sqrt{d}}, \frac{m+N}{\sqrt{d}}\right) \\
& \leq \frac{1}{2 N} \sum_{m=-N}^{N} \frac{\sqrt{8}\left\|\gamma^{\prime}\right\|_{\infty}}{\pi \operatorname{dist}(x, \Gamma)} C h^{2} 2 \\
& =\frac{\sqrt{8}\left\|\gamma^{\prime}\right\|_{\infty} C}{\pi \operatorname{dist}(x, \Gamma)} h^{3}(2 N+1) .
\end{aligned}
$$

With $2 N+1 \leq 3 N$ the assertion is proved.

\subsection{Convergence in the second step}

For $x \in G$ and $i, k \in\{1,2\}$ the function $t \mapsto k_{i k}(x, t)$ is continuously differentiable in $[-1,1]$. So there exists the constant

$$
L(x):=\max _{i, k \in\{1,2\}} \max _{t \in[-1,1]}\left|\partial_{t} k_{i k}(x, t)\right|
$$

which is needed for the next estimate. 
Lemma 2. For $F_{2}(x, d, h)$ we have

$$
F_{2}(x, d, h) \leq \frac{6 \sqrt{2}\|u\|_{\infty} L(x)}{\sqrt{\pi}} \sqrt{d} h,
$$

where the constant $L(x)$ is defined in (7).

Proof. For $t, m h \in[-1,1]$ and $i, k \in\{1,2\}$ by the mean value theorem we get

$$
\left|k_{i k}(x, t)-k_{i k}(x, m h)\right| \leq L(x)|t-m h|
$$

This leads to the following estimate for the components of $\Psi_{d, h}-\Phi_{d, h}$ :

$$
\begin{aligned}
\left|\left(\Psi_{d, h}\right)_{i}(x)-\left(\Phi_{d, h}\right)_{i}(x)\right| \leq & \frac{\left\|u_{1}\right\|_{\infty} L(x)}{\sqrt{\pi d}} \sum_{m=-N}^{N} \int_{-1}^{1}|t-m h| e^{-\frac{(t-m h)^{2}}{d h^{2}}} d t \\
& +\frac{\left\|u_{2}\right\|_{\infty} L(x)}{\sqrt{\pi d}} \sum_{m=-N}^{N} \int_{-1}^{1}|t-m h| e^{-\frac{(t-m h)^{2}}{d h^{2}}} d t \\
\leq & \frac{2\|u\|_{\infty} L(x)}{\sqrt{\pi d}} \sum_{m=-N}^{N} \int_{-1}^{1}|t-m h| e^{-\frac{(t-m h)^{2}}{d h^{2}}} d t .
\end{aligned}
$$

Using

$$
\begin{aligned}
\int_{-1}^{1}|t-m h| e^{-\frac{(t-m h)^{2}}{d h^{2}}} d t & =\frac{d}{N^{2}}\left(1-\frac{1}{2} e^{-\frac{1}{d}(m-N)^{2}}-\frac{1}{2} e^{-\frac{1}{d}(m+N)^{2}}\right) \\
& \leq \frac{d}{N^{2}}
\end{aligned}
$$

we obtain

$$
\begin{aligned}
\left|\left(\Psi_{d, h}\right)_{i}(x)-\left(\Phi_{d, h}\right)_{i}(x)\right| & \leq \frac{2\|u\|_{\infty} L(x)}{\sqrt{\pi d}} \sum_{m=-N}^{N} \frac{d}{N^{2}} \\
& =\frac{2\|u\|_{\infty} L(x)}{\sqrt{\pi d}} \frac{d(2 N+1)}{N^{2}}
\end{aligned}
$$

With

$$
F_{2}(d, h, x) \leq \sqrt{2} \max _{i=1,2}\left|\left(\Psi_{d, h}\right)_{i}(x)-\left(\Phi_{d, h}\right)_{i}(x)\right|
$$

we finally get

$$
F_{2}(d, h, x) \leq \frac{6 \sqrt{2}\|u\|_{\infty} L(x)}{\sqrt{\pi}} \sqrt{d} h
$$

as asserted. 
Remark. We notice that the error $F_{2}$ is getting small if the term $\sqrt{d} h$ is getting small, this means if the Gaussian kernel

$$
t \mapsto \frac{e^{-\frac{(t-m h)^{2}}{d h^{2}}}}{\sqrt{\pi d}}
$$

is a good approximation to the Dirac delta function concentrated in the point $m h$. Considering this background, the second approximation step is quite natural.

\subsection{Convergence in the first step}

For $m \in\{-N, \ldots, N\}$ we consider the linear spline

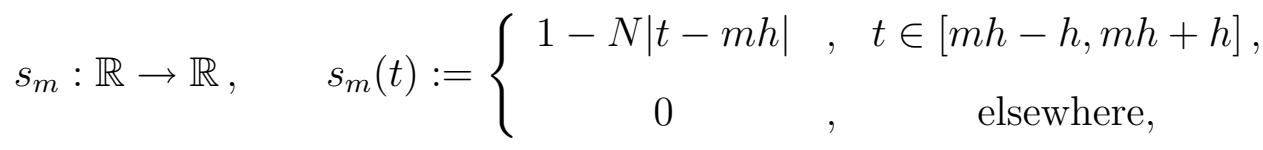

and define the spline interpolants

$$
u_{i, h}^{s p}:[-1,1] \rightarrow \mathbb{R}, \quad u_{i, h}^{s p}(t):=\sum_{m=-N}^{N} u_{i}(m h) s_{m}(t), \quad i=1,2
$$

and

$$
u_{h}^{s p}:[-1,1] \rightarrow \mathbb{R}^{2}, \quad u_{h}^{s p}(t):=\left(\begin{array}{c}
u_{1, h}^{s p}(t) \\
u_{2, h}^{s p}(t)
\end{array}\right)=\sum_{m=-N}^{N} u(m h) s_{m}(t) .
$$

Since $u \in C^{2}\left([-1,1], \mathbb{R}^{2}\right)$ we have $u_{1}, u_{2} \in C^{2}([-1,1])$ and hence there hold the estimates $[7]$

$$
\left\|u_{i}-u_{i, h}^{s p}\right\|_{\infty} \leq \frac{1}{8}\left\|u_{i}^{\prime \prime}\right\|_{\infty} h^{2}, \quad i=1,2 .
$$

Using the linear spline interpolant instead of approximate approximations in the first and second approximation step, we obtain

$$
\begin{aligned}
\Psi_{h}^{s p}(x) & :=\int_{-1}^{1} K(x, t) u_{h}^{s p}(t) d t \\
& =\sum_{m=-N}^{N} \int_{-1}^{1} K(x, t) u(m h) s_{m}(t) d t
\end{aligned}
$$

and

$$
\begin{aligned}
\Phi_{h}^{s p}(x) & :=\sum_{m=-N}^{N} K(x, m h) u(m h) \int_{-1}^{1} s_{m}(t) d t \\
& =\frac{1}{2 N} \sum_{m=-N}^{N} K(x, m h) u(m h)\left(2-\delta_{|m| N}\right),
\end{aligned}
$$


respectively.

The next lemma shows a remarkable convergence result if the parameter $d$ tends to zero.

Lemma 3. For all $x \in G$ we have

$$
\lim _{d \rightarrow 0} \Phi_{d, h}(x)=\Phi_{h}^{s p}(x)
$$

Proof. Using

$$
\lim _{d \rightarrow 0} \operatorname{erf}\left(\frac{m-N}{\sqrt{d}}, \frac{m+N}{\sqrt{d}}\right)=\lim _{d \rightarrow 0} \frac{2}{\sqrt{\pi}} \int_{\frac{m-N}{\sqrt{d}}}^{\frac{m+N}{\sqrt{d}}} e^{-t^{2}} d t
$$

and

$$
\frac{2}{\sqrt{\pi}} \int_{0}^{\infty} e^{-t^{2}} d t=1
$$

we get

$$
\lim _{d \rightarrow 0} \operatorname{erf}\left(\frac{m-N}{\sqrt{d}}, \frac{m+N}{\sqrt{d}}\right)=\left\{\begin{array}{lc}
1, & m=-N \\
2, & |m| \neq N \\
1, & m=N
\end{array}\right.
$$

hence

$$
\lim _{d \rightarrow 0} \operatorname{erf}\left(\frac{m-N}{\sqrt{d}}, \frac{m+N}{\sqrt{d}}\right)=2-\delta_{|m| N}
$$

This implies

$$
\begin{aligned}
\lim _{d \rightarrow 0} \Phi_{d, h}(x) & =\lim _{d \rightarrow 0}\left(\frac{1}{2 N} \sum_{m=-N}^{N} K(x, m h) u(m h) \operatorname{erf}\left(\frac{m-N}{\sqrt{d}}, \frac{m+N}{\sqrt{d}}\right)\right) \\
& =\frac{1}{2 N} \sum_{m=-N}^{N} K(x, m h) u(m h) \lim _{d \rightarrow 0} \operatorname{erf}\left(\frac{m-N}{\sqrt{d}}, \frac{m+N}{\sqrt{d}}\right) \\
& =\frac{1}{2 N} \sum_{m=-N}^{N} K(x, m h) u(m h)\left(2-\delta_{|m| N}\right) \\
& =\Phi_{h}^{s p}(x),
\end{aligned}
$$

as asserted.

With help of the above convergence result, the remaining error in the first approximation step can be controlled, too.

Lemma 4. Let $x \in G$. For every $\varepsilon>0$ there exists some $d_{0}>0$ such that for all $d \leq d_{0}$ we have

$$
F_{1}(d, h, x) \leq \varepsilon+4\|u\|_{\infty} L(x) h+\frac{6 \sqrt{2}\|u\|_{\infty} L(x)}{\sqrt{\pi}} \sqrt{d} h+\frac{\left\|\gamma^{\prime}\right\|_{\infty}\left\|u^{\prime \prime}\right\|_{\infty}}{\pi \operatorname{dist}(x, \Gamma)} h^{2} .
$$


Proof. Choose $\varepsilon>0$. Due to the last Lemma there exists some $d_{0}>0$ such that for all $d \leq d_{0}$ the estimate

$$
\left|\Phi_{h}^{s p}(x)-\Phi_{d, h}(x)\right| \leq \varepsilon
$$

holds. Using the inequatities (4), (8) and $\left\|u_{i}\right\|_{\infty} \leq\|u\|_{\infty}$ we get

$$
\begin{aligned}
\left|v(x)-\Psi_{h}^{s p}(x)\right| & =\left|\int_{-1}^{1} K(x, t)\left(u(t)-u_{h}^{s p}(t)\right) d t\right| \\
& \leq \frac{1}{2 \pi} \int_{-1}^{1}\left|K(x, t)\left(u(t)-u_{h}^{s p}(t)\right)\right| d t \\
& \leq \int_{-1}^{1} 4 \frac{\left\|\gamma^{\prime}\right\|_{\infty}}{\pi \operatorname{dist}(x, \Gamma)} \frac{1}{8}\left\|u^{\prime \prime}\right\|_{\infty} h^{2} d t \\
& =\frac{\left\|\gamma^{\prime}\right\|_{\infty}\left\|u^{\prime \prime}\right\|_{\infty}}{\pi \operatorname{dist}(x, \Gamma)} h^{2} .
\end{aligned}
$$

Furthermore we find

$$
\begin{aligned}
\left|\Psi_{h}^{s p}(x)-\Phi_{h}^{s p}(x)\right| & \leq \sum_{m=-N}^{N} \int_{-1}^{1}|(K(x, t)-K(x, m h)) u(m h)| s_{m}(t) d t \\
& \leq 4\|u\|_{\infty} L(x) \sum_{m=-N}^{N} \int_{-1}^{1}|t-m h| s_{m}(t) d t \\
& \leq 4\|u\|_{\infty} L(x) \sum_{m=-N}^{N} \frac{h^{2}}{3} . \\
& \leq 4\|u\|_{\infty} L(x) h,
\end{aligned}
$$

where $L(x)$ ist the constant defined in (7). Using the decomposition

$$
\begin{aligned}
F_{1}(d, h, x) \leq & \left|v(x)-\Psi_{h}^{s p}(x)\right|+\left|\Psi_{h}^{s p}(x)-\Phi_{h}^{s p}(x)\right| \\
& +\left|\Phi_{h}^{s p}(x)-\Phi_{d, h}(x)\right|+\left|\Phi_{d, h}(x)-\Psi_{d, h}(x)\right|
\end{aligned}
$$

and the convergence result from the second step, we obtain the assertion.

Now collecting the estimates from all the three steps, the following main estimate is proved:

Theorem. Let $x \in G$. For every $\varepsilon>0$ there exists some $d_{0}>0$ such that for all $d \leq d_{0}$ we have

$$
\begin{aligned}
F(x, d, h) \leq & \varepsilon+4\|u\|_{\infty} L(x) h+\frac{6 \sqrt{2}\|u\|_{\infty} L(x)}{\sqrt{\pi}} \sqrt{d} h \\
& +\frac{\left\|\gamma^{\prime}\right\|_{\infty}}{\pi \operatorname{dist}(x, \Gamma)}\left(3 \sqrt{8} C+\left\|u^{\prime \prime}\right\|_{\infty}\right) h^{2}
\end{aligned}
$$

where $C$ is the constant from $(5)$, and $L(x)$ is defined in $(7)$. 


\section{References}

[1] W. Borchers, Über das Anfangs-Randwertproblem der instationären StokesGleichung, Z.A.M.M. 65, T329-T330, 1985.

[2] G.B. Folland, Introduction to Partial Differential Equations, Princeton University Press, 1995.

[3] W. Hackbusch, Integralgleichungen, Teubner, 1989.

[4] G.C. Hsiao, Integral Representation of Solutions for Two-Dimensional Viscous Flow Problems, Integral Equations and Operator Theory 5, 533-547, 1982.

[5] T. Ivanov, Boundary Layer Approximate Approximations and Cubature of Potentials in Domains, Linköping Studies in Science and Technology, Theses No. 516, Linköping University, 1997.

[6] S. König, Experimentelle Konvergenzordnung einer Randpunktmethode, Diplomarbeit, Universität Kassel Fachbereich Mathematik/Informatik, 2003.

[7] R. Kress, Linear Integral Equations, Springer, 1989.

[8] O.A. Ladyzhenskaya, The Mathematical Theory of Viscous Incompressible Flow, Gordon and Breach, New York et al., 1969.

[9] V. Maz'ya, A New Approximation Method and its Applications to the Calculation of Volume Potentials, Boundary Point Method, in: 3. DFG-Kolloquium des DFGForschungsschwerpunktes Randelementmethoden, 30.09.-05.10.1991.

[10] V. Maz'ya, Boundary Point Method, LITH-MATH-R-91-44, Dept. of Math., Linköping University, 1991.

[11] V. Maz'ya, G. Schmidt, On Approximate Approximations using Gaussian Kernels, IMA J. Num. Anal. 16, 13-29, 1996.

[12] F. Müller, W. Varnhorn: An Approximation Method using Approximate Approximations. Applic. Anal. 85 (2006) 669 - 680

[13] F. Müller, W. Varnhorn: Error Estimates for Approximate Approximations on Compact Intervalls. J. Approx. T. 145 (2007) 171 - 181

[14] F.K.G. Odquist, Über die Randwertaufgaben in der Hydrodynamik zäher Flüssigkeiten, Math. Z. 32, 329-375, 1930.

[15] A.N. Popov, Application of Potential Theory to the Solution of a Linearized System of Navier-Stokes Equations in the Two-Dimensional Case, Proceedings of the Steklov Institute of Mathematics 116 (Ed.: O.A. Ladyzhenskaya), 167-186, Providence R.I., AMS, 1973.

[16] H.R. Schwarz, Numerische Mathematik, Teubner, 1997.

[17] W. Varnhorn, The Stokes Equations, Mathematical Research Vol. 76, Akademie Verlag, 1994.

[18] D. Werner, Funktionalanalysis, Springer, 1995. 